\title{
Rapid and sensitive real-time assay for the detection of respiratory syncytial virus using RT-SIBA ${ }^{\circledast}$
}

Kevin E. Eboigbodin ${ }^{1 *}$, Kirsi Moilanen $^{1 \dagger}$, Sonja Elf ${ }^{1}$ and Mark Hoser ${ }^{2}$

\begin{abstract}
Background: Respiratory syncytial virus (RSV) is one of the most common causes of respiratory tract infections among young children and the elderly. Timely and accurate diagnosis of respiratory tract infections improves patient care and minimizes unnecessary prescriptions of antibiotics. We sought to develop a rapid nucleic acid tests for the detection of RSV within minutes, while retaining the high sensitivity achieved with RT-PCR.

Methods: We developed and evaluated a reverse transcription isothermal nucleic acid amplification method, reverse transcription strand invasion based amplification (RT-SIBA), for the rapid detection of RSV.

Results: The developed RT-SIBA assay showed good sensitivity by detecting as few as 10 copies of RSV RNA within 20 min compared with reverse transcription polymerase chain reaction, which took approximately $2 \mathrm{~h}$. The performance of the RT-SIBA RSV assay was further investigated using nasopharyngeal swab specimens. The RT-SIBA assay had a sensitivity of $100 \%(25 / 25)$ and a specificity of 100\% (15/15).

Conclusion: RT-SIBA did not require highly purified RNA for the rapid detection of RSV and was therefore compatible with rapid specimen processing methods. This reduces the complexity of specimen preparation and further shortens the total amount of time needed to detect RSV in clinical specimens. The developed RT-SIBA assay for RSV could be a useful tool for prompt management of this infection
\end{abstract}

\section{Background}

Respiratory syncytial virus (RSV) is a leading cause of respiratory tract infections and is often associated with high morbidity and mortality rates among children as well as the elderly and immunocompromised patients $[1,2]$. RSV accounts for more than $60 \%$ of acute respiratory tract infection cases among young children. For children younger than 1 year, RSV can account for approximately $80 \%$ of lower respiratory infections, and almost all children are infected by RSV before the age of 3 years [3, 4]. RSV is a member of the Paramyxoviridae family and harbors non-segmented negative-sense singlestranded RNA as its genetic material. There are two major RSV subtypes, RSV-A and RSV-B, which are distinguished by their surface glycoproteins and genetic variation [5].

\footnotetext{
* Correspondence: kevin.eboigbodin@oriondiagnostica.fi

${ }^{\dagger}$ Equal contributors

${ }^{1}$ Research and Development, Orion Diagnostica Oy, P. O. BOX 83FI-02101

Espoo, Finland

Full list of author information is available at the end of the article
}

The two RSV subtypes often co-circulate during epidemic seasons $[6,7]$.

Timely and accurate diagnosis of RSV infections plays an important role in their management and treatment because it is often challenging to distinguish RSV infections from other viral or bacterial respiratory tract infections based on the clinical presentation alone [8]. Timely diagnosis also plays a crucial role in avoiding inappropriate use of antibiotics, thereby lowering the risk of antimicrobial resistance [9]. Nucleic acid amplification tests (NAATs), especially reverse transcription polymerase chain reaction (RT-PCR), are increasingly used to detect RSV because they are more sensitive than rapid antigen detection tests (RADTs) [10]. Despite the superior sensitivity of RT-PCR over RADTs, RADTs remain an important diagnostic tool, especially in point-of-care settings, due to their shorter turnaround time and ease of use $[11,12]$. Conversely, specialised training and sophisticated and costly instrumentation are required to 
perform RT-PCR. Consequently, the method is often confined to specialised and large central laboratories.

In this study, we sought to develop a NAAT for RSV that has a similar time-to-detection as RADTs, while retaining the high sensitivity achieved with RT-PCR. To reduce the diagnostic time, we developed an isothermal reverse transcription strand invasion based amplification (RT-SIBA) assay for the detection of RSV. In RT-SIBA reactions, RSV RNA is first transcribed to cDNA by a reverse transcriptase and immediately amplified and detected under isothermal reaction conditions [13, 14]. SIBA utilize a recombinase-coated single-stranded invasion oligonucleotide (IO) for the separation of a target duplex. This leads to the generation of a single-stranded target template, which subsequently becomes bound and extended by target-specific primers via the action of polymerase. The repeated cycles of recombinase-dependent strand separation and subsequent polymerase-dependent extension of the target leads to an exponential amplification under isothermal conditions.

\section{Methods}

\section{Clinical specimens and rapid processing}

A total of 40 nasopharyngeal (NP) swab specimens were obtained from Discovery Life Sciences Biobank (Discovery Life Sciences, Inc., CA, USA). The specimens were previously determined to be positive $(n=25)$ or negative $(n=15)$ for RSV using the Alere BinaxNOW RSV , a rapid immunoassay test (Alere Inc, USA). These specimens were used to evaluate the performance of RT-SIBA in comparison with previously published RT-PCR for the detection of RSV [15]. Viral RNA was extracted from the specimens using the QIAamp Viral RNA Mini Kit (Qiagen, Germany) according to the manufacturer's instructions. Thereafter, $2 \mu \mathrm{l}$ of purified RNA was added to the RT-SIBA or RT-PCR RSV assay. A set of NP swab specimens was also subjected to a quick and crude specimen preparation protocol instead of RNA purification. To this end, $5 \mu \mathrm{l}$ of the NP swab specimen was added to $45 \mu \mathrm{l}$ of lysis buffer (2\% Triton X-100 and $125 \mathrm{mM}$ magnesium acetate; Sigma-Aldrich, USA), and $2 \mu \mathrm{l}$ of the crude lysate was subsequently added to the RT-SIBA reaction.

\section{Preparation of RSV RNA standards}

RNA was purified from RSV-A (ATCC VR-1540) and RSV-B (ATCC VR-1400) American Type Culture Collection (LGC Standards, Germany) viral particles using the QIAamp Viral RNA Mini Kit (Qiagen) according to the manufacturer's instructions. The purified RNA was quantified using the Genesig RSV RT-PCR kit (Primerdesign $^{\text {tw }}$ Ltd., UK). The kit includes RSV RNA standards with pre-determined copy numbers. These standards were used to generate standard curves for the quantification of RSV-A RNA and RSV-B RNA. RT-PCR was performed on a Bio-Rad qPCR CFX95 instrument (Bio-Rad Laboratories, CA, USA). The quantified RSV RNA was subsequently used to establish the analytical sensitivity of the RT-SIBA RSV assay in comparison with a previously published RT-PCR assay for the detection of RSV [15].

\section{Design of the RT-SIBA RSV assay}

The assay was designed to detect all known human RSV strains including subtypes A and B [14]. RSV sequences were retrieved from the Virus Pathogen Resource [16] as well as the National Center for Biotechnology Information database and aligned. The assay was designed to amplify $\sim 70$ nucleotides within the highly conserved region of the RSV N-protein gene. The assay used the same target region within the RSV-A and RSV-B genomes. The RSV-A and RSV-B genomes share about $90 \%$ homology within the chosen target region. A set of primers and invasion oligonucleotides (IOs) were designed to amplify this target region. The RSV assay used one forward primer, one reverse primer, two IOs, and one probe. The use of multiple IOs facilitated improved detection of RSV-A and RSV-B in the same reaction tube, but could not differentiate between the two subtypes of RSV. The RT-SIBA assay was also developed to detect the bacteriophage MS2. Target-specific probes for both the RSV and MS2 assays were designed as previously described [17]. This allowed the RSV and MS2 assays to be multiplexed in the same reaction tube. The MS2 assay served as an internal control for monitoring sample-derived inhibition [18].

\section{RT-SIBA RSV assay}

RT-SIBA reactions were performed using the SIBA $^{\circ}$ reagent kit (Orion Diagnostica Oy, Finland) with the addition of 16 units of GoScript ${ }^{\text {tw }}$ Reverse Transcriptase (Promega, UK). The RSV forward and reverse primers were each used at a concentration of $400 \mathrm{nM}$. RSV-A, RSV-B, and MS2 IOs were used at concentrations of 200, 200, and $100 \mathrm{nM}$, respectively. The RSV and MS2 probes were each used at a concentration of $100 \mathrm{nM}$. The sequences of the oligonucleotides used for the RT-SIBA RSV and MS2 assays are shown in Table 1. In total, 2000 copies of MS2 RNA (Sigma-Aldrich) were added to the reaction. The UvsX and gp32 enzymes were used at a concentration of $0.25 \mathrm{mg} / \mathrm{ml}$. In total, $2 \mu \mathrm{l}$ of the sample or RNA template was used in a total reaction volume of $20 \mu \mathrm{l}$. RT-SIBA was detected using fluorophore-labeled probes and SYBR Green 1 (1:100,000 dilution). The reactions were incubated at $41{ }^{\circ} \mathrm{C}$ for $60 \mathrm{~min}$, and fluorescence readings were taken at $60 \mathrm{~s}$ intervals using Bio-Rad CFX 96 (Bio-Rad Laboratories). A melting curve profile was generated after amplification at $40-95{ }^{\circ} \mathrm{C}$ to verify that the reaction products were specific. 
Table 1 RT-SIBA oligonucleotides used in this study

\begin{tabular}{|c|c|}
\hline Name & Sequence $5^{\prime}->3^{\prime}$ \\
\hline RSV-F primer & GAGCCACCTCTCCCAT \\
\hline RSV-R primer & AGTCAACATTGAGATA \\
\hline RSV-A IO & CCCCC TITCTITAGCATTITITGTAGGATTT mUmCmUmAmG mAmUmUmCmUmA \\
\hline RSV-B IO & CCCCC TCTCTITAGCATTITITGTAGGACTT mUmCmUmAmG mAmUmUmCmUmA \\
\hline RSV probe & /56-ROXN/+CA + A + TA + T+T+GA+GA+TA/3IABkFQ/ \\
\hline MS2-F primer & CATGATATTCTGGGCAATAGT \\
\hline MS2-R primer & CGTAGATGCCTATGGTTC \\
\hline MS2 IO & CCCCCCCCCC AATAGTCAAATCGACCCAAATCCATTTT mGmGmUmAmA mCmGmCmCmGmGmAmAmC \\
\hline MS2 probe & $/ 5 C Y 5 / A+T A+T T+C T+G G+G C+A+A / 3 \mid A b R Q S p /$ \\
\hline
\end{tabular}

\section{RT-qPCR}

The performance of the RT-SIBA assay was compared with a previously published RT-PCR assay for the detection of RSV-A and RSV-B [15]. Both RT-SIBA and RTPCR reactions were performed at the same time. $2 \mu \mathrm{l}$ of the sample or RNA template was used in a total RTPCR reaction volume of $20 \mu \mathrm{l}$. The concentrations of the probes and primers were previously described [15]. RTPCR was performed using EXPRESS qPCR SuperMix (Thermo Fisher Scientific, USA). A thermal program of $50{ }^{\circ} \mathrm{C}$ for $15 \mathrm{~min}, 95^{\circ} \mathrm{C}$ for $2 \mathrm{~min}$, and 45 cycles of $95{ }^{\circ} \mathrm{C}$ for $30 \mathrm{~s}$ and $45^{\circ} \mathrm{C}$ for $1 \mathrm{~min}$ was used with Bio-Rad qPCR equipment (Bio-Rad Laboratories).

\section{Results}

\section{Analytical sensitivity of the RT-SIBA RSV assay in compari-} son with RT-PCR

The analytical sensitivity of the RT-SIBA RSV assay was compared with that of the previously published RT-PCR RSV assay [15]. The sensitivities of the RTSIBA and RT-PCR assays for RSV were evaluated in three independent experiments by adding serial dilutions of RNA extracted from RSV, with $10-10^{5}$ copies per reaction. Five replicates of each dilution were used. RT-SIBA reactions were detected using both target-specific probes and SYBR Green. The RSV assay included the MS2 internal control to assess sample-derived inhibition. The RSV probe was labeled with ROX and Iowa Black FQ quencher, whereas the MS2 probe was labeled with Cy5 and Iowa Black RQ quencher.

RT-PCR incorporated a target-specific probe, which enabled specific detection of RSV-A and RSV-B subtypes. The results are shown in Fig. 1 and Table 2 . Both RT-SIBA and RT-PCR reliably detected as few as 10 copies of RSV RNA (5/5 replicates in every control dilution). The average amount of time taken to achieve positive reactions in RT-SIBA was compared with the corresponding cycle threshold (ct) values for RTPCR (Table 2). RT-SIBA reactions were performed at a constant temperature and consequently did not require thermal cycling. Hence, RT-SIBA data were collected at $1 \mathrm{~min}$ intervals. RT-SIBA RSV assays detected 100 copies of RSV-A and RSV-B at approximately 14 and $16 \mathrm{~min}$, respectively. The corresponding ct values for 100 copies of RSV-A and RSV-B RNA were approximately 27 and 30, respectively. The corresponding average amount of time taken to achieve positive reactions with RT-PCR can be around $2 \mathrm{~h}$ due to the initial reverse transcription step as well as the instrument ramp time. RSV was detected faster by RT-SIBA than by RT-PCR.

\section{Analytical specificity of the RT-SIBA RSV assay}

Subsequent melting curve analysis revealed the presence of a single specific amplicon in reactions

Table 2 Analytical sensitivity and average detection time of RT-SIBA compared with those of RT-PCR for the detection of RSV

\begin{tabular}{|c|c|c|c|c|}
\hline \multirow[b]{2}{*}{$\begin{array}{l}\text { RSV } \\
\text { RNA copy number } \\
\text { per reaction }\end{array}$} & \multicolumn{2}{|c|}{$\begin{array}{l}\text { RT-SIBA } \\
\text { Average amount of time } \\
\text { taken to achieve positive } \\
\text { results (minutes) }\end{array}$} & \multicolumn{2}{|c|}{$\begin{array}{l}\text { RT-PCR } \\
\text { Average cycle } \\
\text { threshold to achieve } \\
\text { positive results (ct) }\end{array}$} \\
\hline & RSV-A & RSV-B & RSV-A & RSV-B \\
\hline $10^{4}$ & 11 & 13 & 21 & 20 \\
\hline $10^{3}$ & 12 & 14 & 24 & 23 \\
\hline $10^{2}$ & 14 & 16 & 27 & 26 \\
\hline $10^{1}$ & 16 & 20 & 31 & 30 \\
\hline 0 & ND & ND & ND & ND \\
\hline
\end{tabular}

$N D$ not determined, $R S V$ respiratory syncytial virus, $R T$-PCR reverse transcription polymerase chain reaction, $R T$-SIBA reverse transcription strand invasion based amplification 


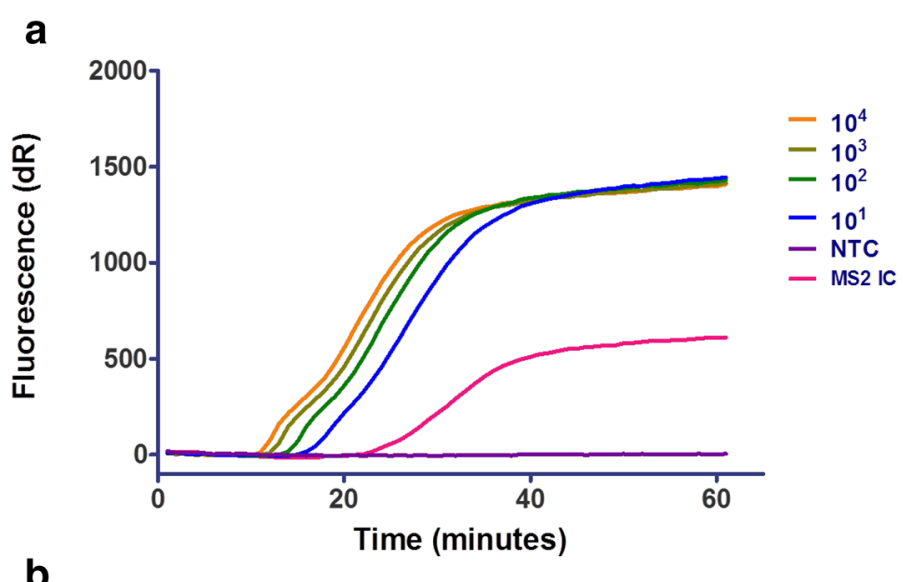

b

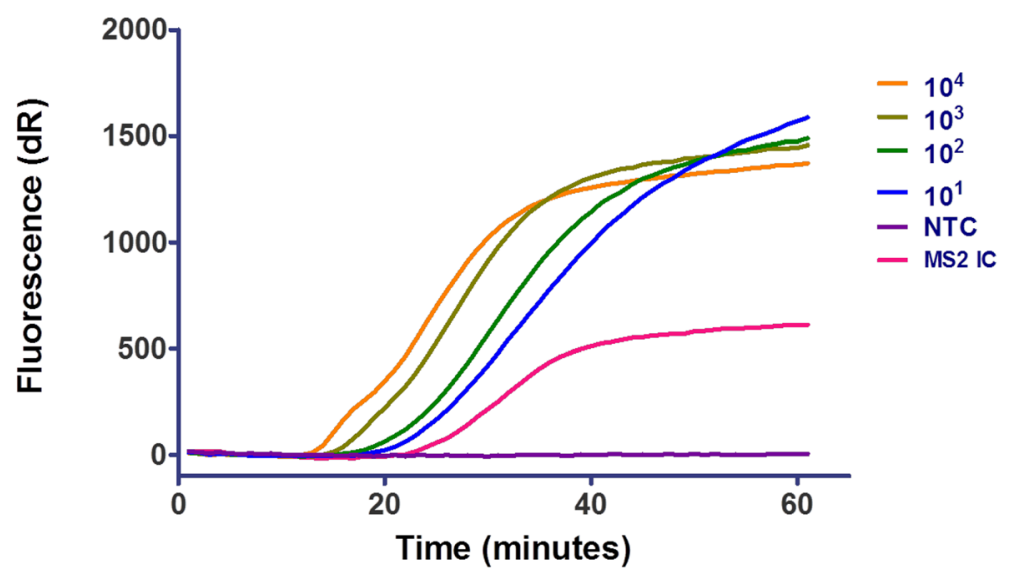

C

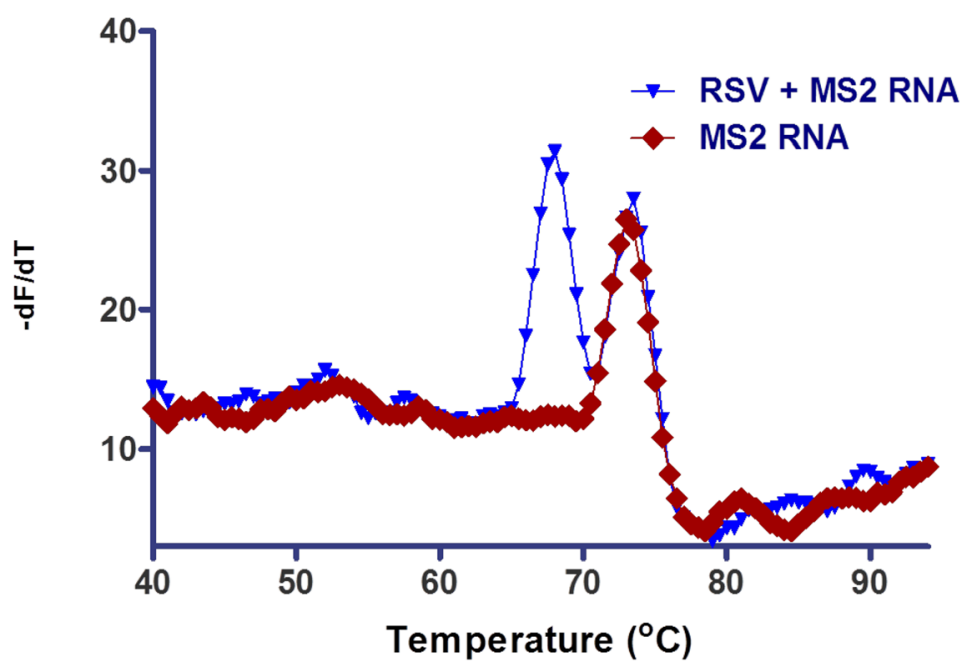

Fig. 1 Sensitivity of RT-SIBA for the detection of respiratory syncytial virus (RSV). a RSV-A RNA and $\mathbf{b}$ RSV-B RNA. c Melting curve profiles of RSV (1000 copies of RSV-A RNA) and MS2 RNA in the same reaction tube. MS2 was used as the internal control (IC, 2000 copies per reaction). No template control (NTC)

containing RSV RNA. The RT-SIBA RSV assay did not produce any detectable amplification signal in the absence of RSV RNA (no template controls). This was also the case when related nucleic acids from other common respiratory pathogens were added to the RT-SIBA RSV reactions (adenovirus, influenza A, influenza B, rhinovirus, coronavirus, Staphylococcus aureus, and Streptococcus pyogenes). These findings, together with the negative sample panel, showed the specificity of the RT-SIBA RSV assay. 
Performance of RT-SIBA RSV with NP swab specimens The performance of RT-SIBA for the detection of RSV was further evaluated using NP swab specimens. A total of 40 NP specimens were used, 15 of which were negative for RSV and the other 25 were positive for RSV, as previously determined by the Alere BinaxNOW RSV test. Total RNA were extracted from these specimens and subsequently used to further evaluate the performance of the RT-SIBA and RT-PCR RSV assays. The results are shown in Table 3. Both the RT-SIBA and RT-PCR RSV assays detected RSV in all $25 \mathrm{NP}$ specimens that were previously confirmed to be positive for RSV. Conversely, no amplification reaction was detected by the RT-SIBA RSV assay with the $25 \mathrm{NP}$ specimens that were previously confirmed to be negative for RSV. The RT-SIBA RSV assay detected RSV in positive specimens within approximately $20 \mathrm{~min}$, while the detection time of RT-PCR RSV was approximately $2 \mathrm{~h}$. The faster detection time is one advantage of RT-SIBA over RT-PCR.

\section{Rapid specimen processing}

We evaluated the possibility of performing one-step rapid lysis of NP specimens, instead of purifying RNA from them. This reduces the complexity of specimen preparation and could further shorten the total amount of time needed to detect RSV from clinical samples. The results are shown in Table 3. RSV was detected in all 25 RSV-positive NP specimens by RT-SIBA using this crude sample lysis method. The detection of RSV by RT-SIBA was faster with clinical specimens extracted using a commercial extraction kit than with clinical specimens subjected to the rapid sample processing method. This could be due to a difference in the final amount of RNA present in the reaction. RSV was not detected in any of

Table 3 Performance of RT-SIBA and RT-PCR for the detection of RSV from NP swab specimens using purified RNA or the rapid lysis protocol

\begin{tabular}{|c|c|c|c|c|}
\hline \multirow[b]{2}{*}{ Sample no. } & \multirow[b]{2}{*}{ Original sample type } & \multicolumn{2}{|c|}{ Extracted RNA from clinical specimens } & \multirow{2}{*}{$\begin{array}{l}\text { Rapid sample processing } \\
\text { RT-SIBA detection time (minutes) }\end{array}$} \\
\hline & & RT-SIBA detection time (minutes) & RT-PCR, cycle threshold Ct (result) & \\
\hline 1 & NP UTM & 10 & 25 (RSV B+) & 16 \\
\hline 2 & NP UTM & 11 & 27 (RSV B+) & 31 \\
\hline 3 & NP UTM & 11 & 27 (RSV B+) & 19 \\
\hline 4 & NP UTM & 10 & 24 (RSV B+) & 22 \\
\hline 5 & NP UTM & 11 & 26 (RSV B+) & 15 \\
\hline 6 & NP UTM & 11 & 27 (RSV B+) & 17 \\
\hline 7 & NP UTM & 11 & 26 (RSV B+) & 15 \\
\hline 8 & NP UTM & 10 & 23 (RSV B+) & 16 \\
\hline 9 & NP UTM & 11 & 28 (RSV B+) & 44 \\
\hline 10 & NP UTM & 12 & 29 (RSV B+) & 20 \\
\hline 11 & NP UTM & 10 & 23 (RSV B+) & 16 \\
\hline 12 & NP UTM & 20 & 25 (RSV B+) & 36 \\
\hline 13 & NP UTM & 14 & 28 (RSV B+) & 35 \\
\hline 14 & NP UTM & 11 & 28 (RSV B+) & 16 \\
\hline 15 & NP UTM & 12 & 28 (RSV B+) & 20 \\
\hline 16 & NP UTM & 10 & 24 (RSV B+) & 19 \\
\hline 17 & NP UTM & 12 & 26 (RSV A+) & 17 \\
\hline 18 & NP UTM & 11 & 25 (RSV B+) & 21 \\
\hline 19 & NP UTM & 11 & 25 (RSV B+) & 16 \\
\hline 20 & NP UTM & 11 & 33 (RSV B+) & 15 \\
\hline 21 & NP UTM & 20 & 27 (RSV B+) & 37 \\
\hline 22 & NP UTM & 10 & 25 (RSV B+) & 15 \\
\hline 23 & NP VTM & 14 & 31 (RSV A+) & 22 \\
\hline 24 & NP VTM & 12 & 27 (RSV A+) & 15 \\
\hline 25 & NP VTM & 13 & 31 (RSV B+) & 21 \\
\hline $26-40$ & NP UTM & ND & RSV (-) & ND \\
\hline
\end{tabular}


the RSV-negative NP specimens by the RT-SIBA RSV assay. It took approximately $5 \mathrm{~min}$ per specimen to perform the rapid specimen preparation protocol, whereas purification of RNA from NP specimens took approximately $45 \mathrm{~min}$. The results suggest that a crude sample processing protocol could be used with RT-SIBA to further shorten the total amount of time needed to detect RSV from NP specimens.

\section{Discussion}

In this study, we developed and evaluated an alternative NAAT, RT-SIBA, for the rapid detection of RSV from NP swab specimens. This method is reported to be useful for the detection of pathogens [13, 14, 17, 19]. SIBA is an isothermal nucleic acid amplification method that relies on a recombinase-dependent oligonucleotide and target-specific primers to rapidly amplify a DNA duplex $[14,17]$. The inclusion of a reverse transcription enzyme in SIBA reactions (RT-SIBA) facilitates the rapid detection of RNA from pathogens [13]. SIBA reactions are performed at a low and constant temperature $\left(41{ }^{\circ} \mathrm{C}\right)$ and therefore do not require complex instrumentation, in contrast with RT-PCR. Consequently, RT-SIBA is compatible with less complex devices, which could be suitable for point-of-care applications. These portable devices do not require highly specialised personnel to run and interpret the results $[19,20]$. In this study, RTSIBA reactions were performed with a qPCR device for the convenience of performing multiple reaction conditions and replicates. The RT-SIBA assay was developed to detect both the RSV-A and RSV-B subtypes, but not to distinguish between them.

The developed RT-SIBA RSV assay displayed a similar analytical sensitivity and specificity as the RT-PCR method for the detection of RSV. The RT-SIBA assay detected 100 copies of RSV-A and RSV-B RNA after 14 and $16 \mathrm{~min}$, respectively. The detection time differed between the RSV subtypes because the RSV-B target region has two nucleotides that are not complementary to the assay. SIBA can typically tolerate 1-3 nucleotide mutations in the assay target region, but this leads to a slightly slower detection time, as seen with RSV-B RNA [14]. Nonetheless, the detection time reported for RT-SIBA RSV was significantly faster than that of RT-PCR, which took approximately $2 \mathrm{~h}$. RT-SIBA detected RSV in a highly specific manner, without cross-reacting with other common respiratory pathogens.

The performance of the RT-SIBA RSV assay was further verified using $40 \mathrm{NP}$ swab specimens. RT-SIBA detected RSV from all NP specimens that were previously determined to be RSV-positive by RT-PCR. Based on the number of NP samples used, the RT-SIBA assay displayed a sensitivity of $100 \%(25 / 25)$ and a specificity of $100 \%(15 / 15)$. The method was compatible with a rapid sample processing method, further shortening the total amount of time needed to detect RSV from clinical specimens. The detection times of RT-SIBA are similar to those of RADTs. RADTs used to detect RSV can take 15-30 min [21, 22]. However, RADTs are often less sensitive than RT-PCR [10]. Despite the superior sensitivity of RT-PCR over RADTs, RADTs still play an important role in the diagnosis of RSV, especially within point-ofcare settings, due to their rapid detection time and ease of use. RT-SIBA displayed a detection time as rapid as that of RADTs and a high analytical sensitivity similar to that of RT-PCR.

\section{Conclusion}

In conclusion, the developed RT-SIBA for the rapid detection of RSV displayed high levels of sensitivity and specificity for RSV RNA. The method significantly shortens the total detection time needed for the diagnosis of RSV from nasopharyngeal swab specimens. RT-SIBA is compatible with rapid specimen processing, a portable device, and is therefore a useful molecular diagnostics tool for the rapid diagnosis of RSV infections especially within the point of care settings.

\section{Abbreviations}

NAATs: Nucleic acid amplification tests; RADTs: Rapid antigen detection tests; RSV: Respiratory syncytial virus; RT-PCR: Real time polymerase chain reaction; RT-SIBA: Reverse transcription strand invasion based amplification

\section{Acknowledgements}

Not applicable.

\section{Funding}

The work is funded by Orion Diagnostica Oy.

Availability of data and materials

Available upon request.

Authors' contributions

KE conceived the study; KE, KM and SE designed and performed the experiments; $\mathrm{KE}, \mathrm{KM}$ and $\mathrm{SE}$ and $\mathrm{MH}$ analyzed results and wrote the manuscript. All authors read and approved the final manuscript.

\section{Competing interests}

KE, KM, SE are employees of Orion Diagnostica Oy. All SIBA patents/patent applications are owned by Orion Diagnostica Oy. KE, KM, SE and $\mathrm{MH}$ are named inventors in SIBA patents/ patent applications.

Consent for publication

Not applicable.

Ethics approval and consent to participate

Not applicable.

Author details

${ }^{1}$ Research and Development, Orion Diagnostica Oy, P. O. BOX 83FI-02101 Espoo, Finland. ${ }^{2}$ Molecular Biology, GeneForm Technologies, Broadstairs, UK.

Received: 8 December 2016 Accepted: 27 January 2017

Published online: 10 February 2017

References

1. Hall CB. Respiratory syncytial virus and parainfluenza virus. N Engl J Med. 2001;344(25):1917-28. 
2. Falsey AR, Hennessey PA, Formica MA, Cox C, Walsh EE. Respiratory syncytial virus infection in elderly and high-risk adults. N Engl J Med. 2005;352(17):1749-59.

3. Piedimonte G, Perez MK. Respiratory syncytial virus infection and bronchiolitis. Pediatr Rev. 2014;35(12):519-30.

4. Piedimonte G. RSV infections: state of the art. Cleve Clin J Med. 2015; 82(11 Suppl 1):S13-8

5. de-Paris F, Beck C, de Souza Nunes L, Pinheiro AM, Paiva RM, da Silva Menezes D, Pires MR, dos Santos RP, de Souza Kuchenbecker R, Barth AL. Evaluation of respiratory syncytial virus group $A$ and $B$ genotypes among nosocomial and community-acquired pediatric infections in southern Brazil. Virol J. 2014;11(1):1-6.

6. Borchers AT, Chang C, Gershwin ME, Gershwin L. Respiratory syncytial virus-a comprehensive review. Clin Rev Allergy Immunol. 2013;45(3):331-79.

7. Esposito S, Piralla A, Zampiero A, Bianchini S, Di Pietro G, Scala A, Pinzani R, Fossali E, Baldanti F, Principi N. Characteristics and their clinical relevance of respiratory syncytial virus types and genotypes circulating in northern Italy in five consecutive winter seasons. PLoS One. 2015;10(6):e0129369.

8. Thornton HV, Blair PS, Lovering AM, Muir P, Hay AD. Clinical presentation and microbiological diagnosis in paediatric respiratory tract infection: a systematic review. Br J Gen Pract. 2015;65(631):e69-81.

9. Samson LM, Cooke C, MacDonald NE. Analysis of antibiotic use and misuse in children hospitalized with RSV infection. Pediatr Res. 1996;39(S4):111.

10. Mahony JB, Petrich A, Smieja M. Molecular diagnosis of respiratory virus infections. Crit Rev Clin Lab Sci. 2011;48(5-6):217-49.

11. Prendergast C, Papenburg J. Rapid antigen-based testing for respiratory syncytial virus: moving diagnostics from bench to bedside? Future Microbiol. 2013;8(4):435-44.

12. Chartrand C, Tremblay N, Renaud C, Papenburg J. Diagnostic accuracy of rapid antigen detection tests for respiratory syncytial virus infection: systematic review and meta-analysis. J Clin Microbiol. 2015;53(12):3738-49.

13. Eboigbodin K, Filén S, Ojalehto T, Brummer M, Elf S, Pousi K, Hoser M. Reverse transcription strand invasion based amplification (RT-SIBA): a method for rapid detection of influenza A and B. Appl Microbiol Biotechnol. 2016:100(12):5559-67.

14. Hoser MJ, Mansukoski HK, Morrical SW, Eboigbodin KE. Strand Invasion Based Amplification (SIBA ${ }^{\circledast}$ ): a novel isothermal DNA amplification technology demonstrating high specificity and sensitivity for a single molecule of target analyte. PLoS One. 2014;9(11):e112656.

15. Do LAH, van Doorn HR, Bryant JE, Nghiem MN, Van Nguyen VC, Vo CK, Nguyen MD, Tran TH, Farrar J, de Jong MD. A sensitive real-time PCR for detection and subgrouping of human respiratory syncytial virus. J Virol Methods. 2012;179(1):250-5.

16. Pickett BE, Sadat EL, Zhang Y, Noronha JM, Squires RB, Hunt V, Liu M, Kumar S, Zaremba S, Gu Z, et al. ViPR: an open bioinformatics database and analysis resource for virology research. Nucleic Acids Res. 2012;40(D1):D593-8.

17. Eboigbodin KE, Hoser MJ. Multiplex Strand Invasion Based Amplification (mSIBA) assay for detection of Chlamydia trachomatis and Neisseria gonorrhoeae. Sci Rep. 2016;6:20487.

18. Dreier J, Störmer M, Kleesiek K. Use of bacteriophage MS2 as an internal control in viral reverse transcription-PCR assays. J Clin Microbiol. 2005;43(9): 4551-7.

19. Eboigbodin KE, Brummer M, Ojalehto T, Hoser M. Rapid molecular diagnostic test for Zika virus with low demands on sample preparation and instrumentation. Diagn Microbiol Infect Dis. 2016;86(4):369-71.

20. Hirvonen JJ, Matero P, Siebert C et al. Eur J Clin Microbiol Infect Dis. 2016. doi:10.1007/s10096-016-2860-0

21. Jung BK, Choi SH, Lee JH, Lee J, Lim CS. Performance evaluation of four rapid antigen tests for the detection of Respiratory syncytial virus. J Med Virol. 2016;88(10):1720-4.

22. Abels S, Nadal D, Stroehle A, Bossart W. Reliable detection of respiratory syncytial virus infection in children for adequate hospital infection control management. J Clin Microbiol. 2001;39(9):3135-9.

\section{Submit your next manuscript to BioMed Central and we will help you at every step:}

- We accept pre-submission inquiries

- Our selector tool helps you to find the most relevant journal

- We provide round the clock customer support

- Convenient online submission

- Thorough peer review

- Inclusion in PubMed and all major indexing services

- Maximum visibility for your research

Submit your manuscript at www.biomedcentral.com/submit
Biomed Central 